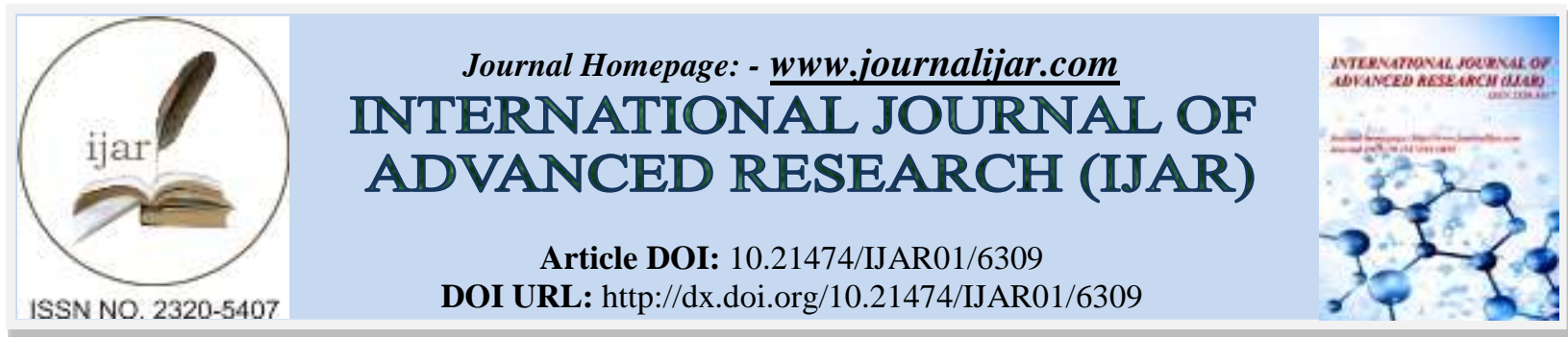

RESEARCH ARTICLE

\title{
INTEGRATION MODEL FOR SCHEDULING OF PASSENGER AND CARGO AIRCRAFT FLIGHT.
}

Desy Widiastuty Alfian and Herman Mawengkang.

Universitas Sumatera Utara.

\section{Manuscript Info}

Manuscript History

Received: 15 November 2017

Final Accepted: 17 December 2017

Published: January 2018

Key words:-

Flight scheduling, NP-Hard.

\begin{abstract}
Nowadays flight scheduling is very important for profitability, but it has a complex problem when compiled traditionally. This study develops an integrated model for scheduling passenger and cargo scheduling simultaneously aiming to maximize profits. Network flow techniques are used to build the model, includes a low flow, the flow of passengers and goods flow. The model is formulated using integer commodity network flow with the characteristics of NP-Hard. Because of the problems predicted a very large, this model is more difficult to solve than the problem of scheduling traditional airlines or cargo. This model is able to directly manage the relationship between passengers and cargo and is expected to be a useful planning tool to determine the appropriate fleet and schedule in the short-term operations
\end{abstract}

Copy Right, IJAR, 2018,. All rights reserved.

\section{Introduction:-}

Effect of economic development led to increased number of passengers or goods using aircraft transportation. At the moment the best flight environment was also used to transport passengers and goods will be introduced here combi flights. In low-combi aircraft as a means of transportation for people and goods is done at the same time. Passenger and freight services are held regularly. Combi flights will provide better benefits than passenger flights or flights of goods (cargo). Therefore, the low-combi need to know the relationship between the passenger and goods so that the use of aircraft and operating performance can be improved. Provisions in the optimization of the system, to consider the linkages between passengers, cargo and combi route flight and flight schedules.

In the 1950s, the airline uses operations research models in solving their complex planning when planning a flight itinerary. Continuous pressure to increase profitability generating more accurate models and better solutions (Klabjan Diego, 2003).

Officers planners determine the passenger and combi into scheduling to be projected to passenger demand, market share, and flight slots at various airports (Ching Hui Tang, 2007). Eligibility check then done, especially the availability of the fleet on route flights, aircraft size, calculation of costs and revenues and fleet crew and aircraft maintenance arrangements. After the flight schedule passenger and combi made, scheduling and cargo flights were made in the plan affected the flight schedule passenger and combi, better still if the projected demand for freight and other constraints related. Improvements can act as feedback to further revise combi flights. This iteration process manually until the desired schedule obtained.

This research will develop a unified scheduling model that combines the passenger and cargo. The goal is to maximize the operating profit. This model is able to directly manage the relationship between passengers and cargo 
and is expected to be a useful planning tool to determine the appropriate fleet and schedule in the short-term operations.

Network flow techniques are used to build the model, includes a low flow, the flow of passengers and goods flow. The model is formulated as a problem IMCN using non characteristic polynomial hard (NP-Hard). Because the problem is expected to be very large, this model is more difficult to solve than the problem of scheduling traditional airlines or cargo.

Here only consider to be served by the low-combi combi aircraft. Although passenger aircraft can transport cargo below the main deck, the inventory space for cargo typically rely on heavy passenger baggage. If the baggage is included in scheduling, capacity of passenger baggage must be the decision variables in the model. In addition, the combi aircraft sometimes allows the seat to be added or removed, implying that the passenger capacity of the cargo may be variable, even though the average capacity of passengers or cargo can be used in the model. That is, the capacity of passengers or cargo on combi aircraft will also be the decision variables in the model.

Yan and Tseng (2004), using the linear integral coupled with network flow multikomoditi. Scheduling of passengers and goods is done separately and not connected in modeling. To resolve the problem always use heuristic methods such as simplex method, engineering branch and bound, cutting plane method, Lagrangian relaxtation, engineering rows of columns and other heuristic methods.

\section{Literature Review:-}

Previous studies about the scheduling of the fleet and scheduling of flights to transport passengers have been carried out by many researchers, such as scheduling model and the routing of the fleet for the transport system (Levin, 1971), a model of scheduling and routing for the flight system (Simpson, 1969), the implementation of the program integer linear assignment problem fleet (Abara, 1989), scheduling and routing airline in the system hub and spokes (Dobson and Lederer, 1993), the assignment problem fleet: troubleshoot integer large scale (Hane etal,1995), maintenance and consideration crew in the fleet assignment (Clark et al, 1996), Yan and Young (1996), Desaulniers etal.(1997), Yan and Tseng (2002), Barnhart etal.(2002), and Lohatepanont and Barnhart (2004). As well as research in freight related has been devoted to matters such as the characteristics of the carrier air express (Chestler, 1985), network planning for an international operator of air cargo (Current et al 1986; Hall, 1989; Lin, 2001; and Lin etal,2003 ), scheduling flights for cargo carriers of air (Okelly, 1986; Antes et al 1998; and Lin \& chen, 2003), competition analysis and analysis of configurations for the operator of air cargo (Lederrer, 1993; Zhang \& Zhang, 2002; and Zhang, 2003 ) and the cargo loading plan for the forwarder (Xue \& Lai, 1997). All this, however, has a different focus of this research, and does not provide a solution at the same passenger cargo scheduling plan.

\section{The model:-}

Mechanicalmultiple time-space network are applied to build a unified scheduling model. This model requires optimal management of the movement of aircraft, passengers and cargo in the network. The main element in the modeling including fleet-flow time-space network, passenger-flow time-space network, and cargo-flow time-space network are described as follows.

\section{The fleet-flow networks time-space-time-space:-}

Some networks are used to formulate the fleet and flight scheduling problems. Each network showed a potential fleet of certain types of movements (passenger fleet, cargo fleet or fleet combi) within a certain time and a certain location. The vertical axis represents the duration of time, while the horizontal axis shows the location of the airport. One dot represents one airport at a certain time, while the arc shows an activity for an airplane. Expressing the arc current flow on the network plane.

There are three types of arcs, namely:

1. Flight leg arc

2. Ground arc

3. Cycle arc

The passenger-flow time-space:-

Networks the time space network technique was also applied to indicate the movement of passengers in accordance with a specific time and location. Each passenger flow space of time representing a pair of couples OD of the 
destination table origin (known as OD table). Such a network is designed to be symmetrical in accordance with the current network fleet of passenger and combi fleet flows, so as to facilitate troubleshooting. There are three types of arcs, namely:

1. Passenger delivery arc,

2. Passenger holding arc,

3. Passenger demand arc.

\section{The cargo-flow time-space networks:-}

Networks cargo flow periods shown in the figure shows the movement of cargo in accordance with the given time and location. According to the time sensitivity of cargo is divided into three kinds of time, one day, four days and seven days (one week). Therefore, unlike the time flow of passengers, cargo flows time flow networks represent a couple of time a specific OD. There are three types of arcs, namely:
1. cargo delivery arc,
2. cargo holding arc,
3. cargo demand arc.

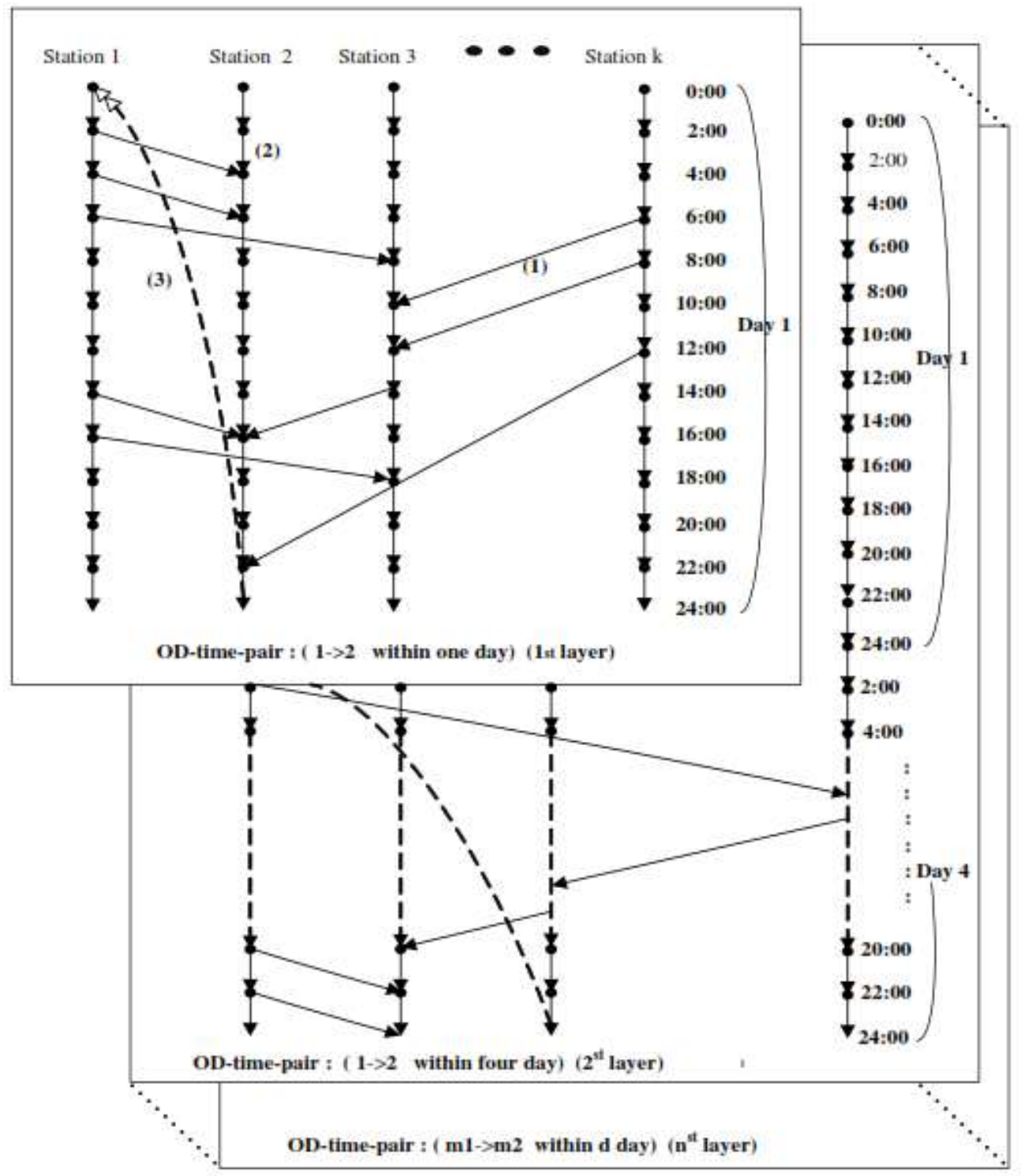

(1) Cargo delivery are (2) Cargo holding arc (3) Cargo demand arc 
In addition to the three main elements, there are several operating constraints that must be considered, including in particular the number of airplanes in each fleet, the quota for each airport and aircraft capacity. In addition the same flight leg in the current fleet of passenger and combi fleet network flow can be served at most one. Similarly, the same flight leg in the current cargo fleet and network flow combi fleet can be served at most one. Flights combi usually added to passenger flights, to set quotas for each airport.

Given the current network fleet, the flow of passengers and cargo as well as the current operational constraints, then the model can be formulated as a mixed integer problem. The purpose of this model is to simultaneously stream of aircraft, passengers and cargo in all the network with minimum cost. Because revenue from passenger traffic and cargo flows including the network in the form of a negative charge, this goal is equivalent to maximize profits.

Here's a list of notations and symbols used in the formulation of the model:

$X_{i j}^{m}, P_{i j}^{l}, Y_{i j}^{n} \quad:$ Current arc $(i, j)$ in the current fleet to-m, to the passenger flow- $l$ and cargo flow to-n;

$C_{i j}^{m}, O_{i j}^{l}, T_{i j}^{n} \quad:$ The cost of arc $(i, j)$ in the current to- $m$ fleet,passenger traffic to- $l$ and cargo flows to-n;

$V_{i}$

$m, M$

$l, L$

: Variable costs at the station $i$ for unity heavy cargo, including loading and unloading cargo;

: Types of fleets to-m;

$n, N$

$A_{m}, N F_{m}$

$D_{l}, N G_{l}$

$B_{n}, N P_{n}$

$A F_{m}$

$F F_{m}, C F_{m}$

$B F_{n}$

$S E^{a b}, S F^{a b}$

: OD pair to-1;

: OD a couple to-n;

: The set of all arcs and points / node on the network stream the fleet to-m;

: The set of all arcs and points / node on the network flow of passengers to-l;

: The set of all arcs and points / node on the network flow of cargo to-n;

: The number of available aircraft in the fleet network to-m;

: The set of all foot bow bow low and low cycle in the network fleet to-m;

: The set of all arcs cargo demand in cargo flows to- $n$ network;

: The set of all foot arc connecting flights station $A$ to station $B$ between the current network of passenger and combi fleet and network current cargo fleet;

$S G^{a}, S H^{a} \quad:$ The set of all flight bow legs associated withstation $a$ in the network flow of passenger and combi fleet and network current cargo fleet;

$Q A^{a b}, Q B^{a b} \quad:$ Quota passenger and cargo airlines agreed that connects the station $A$ to station $\mathrm{B}$;

$Q C^{a}, Q D^{a} \quad$ : Quota passenger and cargo airlines agreed on $a$ to-station;

$\alpha_{i j}, \beta_{i j} \quad:$ The capacity of air passengers in the passenger fleet current network and network combi fleet current (load factor of planning can be used at the planning stage);

$\gamma_{i j}, \delta_{i j}$

$S A, S B$

$S C, S D$

: Network capacity cargo aircraft in the current fleet and network combi cargo fleet current (load factor of planning can be used at the planning stage);

: The set quota airport with passenger flights and cargo flights approved quota.

$U_{i j}^{m}, U_{i j}^{l}, U_{i j}^{n} \quad$ : Flow arc $(i, j)$ flows upward in the current fleet of all $\mathrm{m}$, flow of passengers to- $l$ and cargo flows to-n.

The model is formulated as follows:

The objective function:-

$$
\operatorname{Min} \sum_{m \in M} \sum_{i} C_{i j}^{m} X_{i j}^{m}+\sum_{l \in L} \sum_{i j \in D_{l}} O_{i j}^{l} P_{i j}^{l}+\sum_{n \in N} \sum_{i j \in B_{n}} T_{i j}^{n} Y_{i j}^{n}+\sum_{n \in N} \sum_{i j \in B F_{n}} Y_{i j}^{n}\left(V_{i}+V_{j}\right)
$$

The model is formulated as a mixed integer multiple commodity network flow problem, where the objective is to minimize the function of the system cost, which is equivalent to maximizing profits. In particular, the first term in the objective function is the total cost of the flight, the second term is the total cost of the passenger, the third term is the total cost of freight and the fourth term is the total cost of unloading cargo. There are several obstacles that must be met. Formulations and details of constraints in the developed model is presented as follows.

Flow conservation constraint:-

$$
\sum_{j \in N F_{m}} X_{i j}^{m}-\sum_{k n \in F_{m}} X_{k i}^{m}=0 \quad \forall i \in N F_{m}, \forall m \in M
$$




$$
\begin{aligned}
& \sum_{j \in N G_{l}} P_{i j}^{l}-\sum_{k n \in F_{m}} P_{k i}^{l}=0 \quad \forall i \in N G_{l}, \forall l \in L, \\
& \sum_{j \in N P_{n}} Y_{i j}^{n}-\sum_{k n \in P_{n}} Y_{k i}^{n}=0 \quad \forall i \in N P_{n}, \forall n \in N,
\end{aligned}
$$

constraint (2), (3) and (4) ensure the conservation of flow at every point / vertex in every current fleet, the flow of passengers or cargo flows.

\section{Aircraft constraints:-}

$$
\sum_{i j \in C F_{m}} X_{i j}^{m} \leq A F_{m} \quad \forall m \in M
$$

Constraint (5) shows that the number of aircraft that are used every current network fleet may not exceed the number of available aircraft to the fleet. Airport pair flight quota constraints

Flight leg service constraints:

$$
\begin{aligned}
& \sum_{m \in\{1,2\}} X_{i j}^{m} \leq 1 \quad \forall i j \in F F_{1} \cap F F_{2} \\
& \sum_{m \in\{2,3\}} X_{i j}^{m} \leq 1 \quad \forall i \in F F_{2} \cap F F_{3}
\end{aligned}
$$

Constraints (6) and (7) shows that the same flight leg, diarus fleet of passenger and combi fleet current network as well as network flow combi fleet and current cargo fleet, each at most once.

Airport pair flight quota constraint:

$$
\begin{aligned}
& \sum_{m \in\{1,2\}} \sum_{i j \in S E^{a b}} X_{i j}^{m} \leq Q A^{a b} \quad \forall a b \in S A, \\
& \sum_{i j \in S H^{a}} X_{i j}^{3} \leq Q B^{a b} \quad \forall a b \in S B,
\end{aligned}
$$

Constraint (8) and (9) respectively ensure that the number of all flights in each pair of airports does not exceed the quota of low and low-approved quota.

Airpor flight quota constraint:

$$
\begin{aligned}
& \sum_{m \in\{1,2\}} \sum_{i j \in S G^{a}} X_{i j}^{m} \leq Q C^{a} \quad \forall a \in S C \\
& \sum_{i j \in S H^{a}} X_{i j}^{3} \leq Q D^{a} \quad \forall a \in S D
\end{aligned}
$$

Constraints (10) and (11) respectively ensure that the number of all flights at each station does not exceed the quota of low and low-approved quota.

Aircraft capacity constraints:

$$
\begin{aligned}
& \sum_{l \in L} P_{i j}^{l} \leq\left(x_{i j} X_{i j}^{l}+\beta_{i j} X_{i j}^{2}\right) \quad \forall i j \in F F_{1} \cap F F_{2} \\
& \sum_{m \in N} Y_{i j}^{m} \leq\left(\gamma_{i j} X_{i j}^{2}+\delta_{i j} X_{i j}^{3}\right) \quad \forall i j \in F F_{2} \cap F F_{3}
\end{aligned}
$$

constraint (12) and (13) respectively keep the level of passenger and cargo shipping in the carrying capacity of the aircraft.

Constraints and limits the arc current flow integralitas aircraft:

$$
\begin{aligned}
& 0 \leq X_{i j}^{m} \leq U_{i j}^{m} \quad \forall i j \in A_{m}, \forall m \in M \\
& 0 \leq P_{i j}^{l} \leq U_{i j}^{l} \quad \forall i j \in D_{l}, \forall l \in L \\
& 0 \leq Y_{i j}^{m} \leq U_{i j}^{m} \quad \forall i j \in B_{n}, \forall n \in N
\end{aligned}
$$


constraint (14), (15) and (16) hold all the current arc is in the upper and lower limits.

$X_{i j}^{m} \in$ Integer $\forall i j \in A m, \forall m \in M$

Constraint (17) ensures integralitas air flow.

\section{Conclusions:-}

In this study has developed a model of integration for scheduling of air passengers at cargo flights which aims to minimize operational costs. This model is formulated as a commodity flow problems some integers which belong to the class of NP-Hard. The model is expected to be a useful planning tool for air carriers in deciding the plan scheduling of air passengers at cargo flights, in order to reduce operational costs.

\section{References:-}

1. Abara, J. (1989). Applying integer linear programming to the fleet assignment problem. Interfaces 19, 20-28.

2. Amaruchkul, K. \& Lorchirachoonkul, V. (2011). 'Air-cargo capacity allocation for multiple freight forwarders', Transportation Research Part E: Logistics and Transportation Review 47(1),30-40

3. Antes, J., Campen, L., Derigs, U., Titze, C., Wolle , GD (1998). SYNOPSE: a model-based decision support system for the evaluation of flight schedules for cargo airlines. Decision Support System 22, 307-323.

4. Aziz Zainal. (2016). Nonlinear optimization models for problems with the uncertainty of a cargo container.

5. Barnhart, C., Kniker, TS, Lohatepanont, M. (2002). Itinerary-based airline fleet assignment. Transportation Science 36, 199-217.

6. Chestler, L. (1985). Overnight air express: spatial pattern, competition and the future of small package delivery services. Transportation Quarterly 39, 59-71.

7. Clarke, L., Hane, C., Johnson, EL, and Nemhauser, GL (1996). Maintenance and crew considerations in fl eet assignment. TransportationScience,30, 249-260.

8. Current, JR, Revelle, CS, Cohon, JL (1986). The hierarchical network design problem. European Journal of Operations Research2,57-66.

9. Desaulniers, G., Desrosiers, J., Dumas, Y., Solomon, MM, Soumis, F. (1997). Daily aircraft routing and scheduling.Management Science 43, 841-855. 1997.

10. Dobson, G., Lederer, PJ (1993). Airline scheduling and routing in a hub-and-spoke system. Transportation Science 27, 281-297.

11. Hane, CA, Barnhart, C., Johnson, EL, .Marsten, .R., Nemhauser, GL, Sigismondi, G. (1995). The fleet assignment problem solving a large scale integer programs. Mathematical Study Program 70.211-232.

12. Lederer, PJ (1993). A competition network design problems with pricing. Transportation Science 27, $25-38$.

13. Lin, CC (2001). The freight routing problem of time-de fi nite common delivery freight carriers. Transportation Research Part B 35, 525-547.

14. Lin, CC, Chen, YC (2003). The integration of Taiwanese and Chinese water networks for direct air cargo services. Transportation Research Part A 37, 629-647.

15. Lin, CC Lin, YJ, Lin, DY (2003). The economic effECTS of center-to-center directs on a hub-and-spoke networks for common air express carriers. Journal of Air Transport Management9,255-265.

16. Lohatepanont, M., Barnhart, C. (2004). Airline schedule planning: integrated models and algorithms for design and fl eet schedule assignment. Transportation Science 38, 19-32.

17. Okelly, ME, (1986). The location of interacting hub facilities. TransportationScience.20 (2), 92-106.

18. Rivi Sandhu, Diego Klabjan. (2007). Fleeting with Passenger and Cargo Booking Destination Origin Control. Department of Mechanical and Industrial Engineering University of Illinois at Urbana-ChampaignUrbana, IL.

19. Shangyao Yan and Hwei-FWA Young. (1996). A decision support framework for multi-fleet routing and multistop flight scheduling, Transportation Research Part A 30, 379-398.

20. Simpson, RW (1969). Scheduling and routing models for airline systems. Flight Transportation Laboratory Report R68-3. MIT, Cambridge, Massachusetts.

21. Tang et al. (2008). Transportation Research Part E 44. 1023. 1004-1024

22. Xue, J., Lai, KK (1997). A study on cargo forwarding decision. Computers and Industrial Engineering. 33, 6366.

23. Yan, S., Chen, SC, Chen, CH (2006). Air cargo fl eet routing and timetables setting with multiple on-time demands. Transportation Research Part E 42, 409-430.

24. Zhang, A. (2003). Analysis of an international air-cargo hub: the case of Hong Kong. Journal of Air Transport Management 9, 123-138. 